\title{
EL PROBLEMA DE LA MOTIVACION Y DESARROLLO DEL CAPITAL HUMANO EN LAS PYMES DE ROSARIO, ARGENTINA. UNA PROPUESTA BASADA EN NEUROCIENCIAS
}

\author{
Sergio Albano* \\ Raúl Oviedo** \\ Mariel Santero*** \\ Mirna Sassone ${ }^{\star * * *}$ \\ Silvia Martín*****
}

Resumen. Se presentan los resultados surgidos de una investigación que analiza las múltiples causas de los problemas de motivación del personal vislumbrados en las pequeñas empresas de la ciudad de Rosario, Argentina, y se propone la búsqueda de nuevas alternativas de solución valiéndose de los aportes del conjunto de disciplinas que conforman las Neurociencias. El presente artículo se basa en encuestas de clima laboral realizadas en dos empresas industriales de la ciudad de Rosario, una de las cuales posee dos plantas, involucrando el $100 \%$ del personal operativo. Además, se han realizado entrevistas con el personal directivo y de supervisión. Los resultados así obtenidos se utilizarán para proponer una serie de intervenciones, fundamentadas en el estudio del funcionamiento del cerebro humano, que permitan mejorar la motivación y el rendimiento del personal. A estos estímulos se les dará el nombre de Neuroincentivos.

Palabras Clave: Neuroincentivos; Personal operativo; Clima organizacional.

* Universidad Nacional de Rosario, Argentina.

Contacto: salbano@fcecon.unr.edu.ar

** Universidad Nacional de Rosario, Argentina.

Contacto: roviedo@fcecon.unr.edu.ar

*** Universidad Nacional de Rosario, Argentina.

Contacto: msantero@fcecon.unr.edu.ar

**** Universidad Nacional de Rosario, Argentina.

Contacto: mirnasassone@hotmail.com

***** Universidad Nacional de Rosario, Argentina.

Contacto: silvia2009@express.com.ar 


\title{
THE PROBLEM OF MOTIVATION AND DEVELOPMENT OF HUMAN CAPITAL IN THE PYMES OF ROSARIO, ARGENTINA. A PROPOSAL BASED ON NEUROSCIENCES
}

\begin{abstract}
The results of a research that analyzes the causes of staff motivation problems envisioned in small businesses in the city of Rosario, Argentina, are presented in this article. Further proposes for new alternative solutiona using the contributions of the set of disciplines that make up the Neurosciences are presented. The present article is based on working environment surveys conducted in two industrial companies in the city of Rosario, one of which has two plants, involving $100 \%$ of the operational staff. In addition, interviews with management and supervisory personnel have been made. The results obtained will be used to propose a series of interventions, based on the study of the functioning of the human brain, to improve the staff motivation and performance. These stimuli will be given the name of Neuroincentives.
\end{abstract}

Keywords: Neuroincentives; Operational Staff; Working Enviroment.

Original recibido el 24/05/2018

Aceptado para su publicación el 25/02/2019 


\section{Introducción}

La gestión del capital humano en las PyMEs (pequeñas y medianas empresas) rosarinas presenta múltiples inconvenientes, cuyo principal origen parece ser la falta de motivación y capacitación del personal. Esta problemática, entre otras, comienza a ser abordada por las llamadas Neurociencias, buscando una nueva perspectiva, integradora de los aportes de distintas disciplinas, intentando de este modo hallar nuevas soluciones. Este trabajo se enmarca en el proyecto de investigación ECO188 "Neurociencia como herramienta de motivación y desarrollo del capital humano en las PyMEs rosarinas", acreditado por la Secretaría de Ciencia y Tecnología de la Universidad Nacional de Rosario y desarrollado dentro del Instituto de Investigaciones y Asistencia Tecnológica en Administración (IIATA). Su objetivo principal es "Diseñar un procedimiento experimental para analizar y describir las vinculaciones de la Neurociencia con la motivación y el desarrollo del capital humano, para ser aplicado en dos PyMEs de la ciudad de Rosario".

Además, se propone detectar cuál es la situación actual atinente a las prácticas que llevan a cabo las empresas de la zona en relación con esta temática; indagar en las necesidades de el/la trabajador/a desde la perspectiva de las Neurociencias y analizar las operaciones, funciones y actividades cognoscitivas llevadas a cabo por una persona participe de una organización, con el fin de identificar posibles herramientas de motivación y desarrollo del capital humano.

Se indaga respecto de los conocimientos aportados por las Neurociencias para aplicarlos en el desarrollo de una metodología que permita motivar a las personas que forman parte de una organización, generando un mejor clima laboral y, en definitiva, una mejor calidad de vida para todos los integrantes de la organización.

La aplicación de las neurociencias cognitivas a la conducción de organizaciones permite acceder a nuevos campos de conocimientos para liderar mejor los equipos de trabajo, tomar decisiones con un mayor grado de certeza, capacitar y formar a las personas con técnicas más eficaces, desarrollar acciones comerciales más efectivas y establecer una mejor relación con las personas y el mercado (Braidot, 2014, pág. 19).

Bajo esta nueva óptica, se propone el término Neuroincentivación para aprovechar los aportes de la ciencia y volcarlos al objeto de la investigación.

Se entiende por Neuroincentivación a

la aplicación de los conocimientos provistos por las Neurociencias a la Gestión de Recursos Humanos para mejorar la motivación laboral. La Neuroincentivación implica la provisión de distintos estímulos con el fin de aprovechar al máximo el potencial humano, desarrollando habilidades y destrezas, así como mejorando el clima laboral en general. A estos estímulos se propone denominarlos Neuroincentivos. Los estímulos utilizados desde esta perspectiva son diversos. En muchos casos, son los mismos que venían ya utilizándose hace tiempo, pero a los que ahora las Neurociencias dan fundamento científico. En otros, se trata de herramientas realmente innovadoras. Es a estas últimas que el proyecto apunta y las que serán utilizadas en la experimentación (Albano, Oviedo, Santero, Sassone y Martín, 2016, pág. 6).

Los resultados de estas actividades permitirán conocer cuáles son las actitudes que perjudican, o al menos no colaboran, con el aumento de la productividad. 
Investigando los procesos neuronales que dan origen a estas conductas se podrán seleccionar los Neuroincentivos más apropiados para modificarlas.

La comparación de los indicadores de productividad, eficiencia, ausentismo y accidentes laborales pre y post-experimento determinarán la validez de las hipótesis de trabajo planteadas respecto a la utilidad de los Neuroincentivos aplicados en la intervención.

En caso de resultar satisfactorio el resultado, logrando el involucramiento de los actores, la motivación y el aumento de productividad esperado, con la consiguiente mejora del clima de laboral, podrá establecerse una metodología de trabajo válida para ser aplicada en otras PyMEs de la región.

La búsqueda de antecedentes respecto a este tipo de intervención muestra que, en general, se trabaja sobre los niveles superiores, directivos, pero no el nivel operativo, objeto de este estudio.

La primera etapa del proyecto se dedicó principalmente al relevamiento bibliográfico, la construcción del marco teórico, y la búsqueda de las PyMEs dispuestas a participar del mismo. En una segunda etapa, se trabajó sobre el relevamiento de la situación actual de las mismas, haciendo una medición del clima organizacional de estas empresas, identificando fortalezas y debilidades. A continuación, se profundizó el diagnóstico, complementando el abordaje cuantitativo con focus groups y entrevistas en profundidad, trabajando en paralelo en la planificación de las intervenciones a realizar en cada empresa. Por último, el proyecto cierra con la intervención efectiva en cada empresa y la evaluación de los resultados obtenidos.

El presente trabajo sintetiza los resultados del relevamiento cuantitativo realizado en la segunda etapa del proyecto, teniendo por objetivo evaluar el clima organizacional de cada empresa en estudio, identificando las principales necesidades de intervención para la posterior planificación de los neuroincentivos a aplicar.

\section{Marco teórico}

Gracias a las Neurociencias, y muy especialmente a los avances vertiginosos en ese campo en los últimos diez años, se comprende cómo deviene el cableado eléctrico entre las células neuronales y sus conexiones; y lo que es más importante, se sabe a ciencia cierta que pueden establecerse nuevas conexiones neuronales a partir de las ya existentes desde nuestras vivencias. Esto permite demostrar la plasticidad de nuestras neuronas y, por ende, explicar que el comportamiento humano se basa en ese molde extraordinario que puede cambiar según se regulen las funciones químicas, físicas y psíquicas del ser humano.

Se denomina Neurociencia, en forma genérica, al conjunto de disciplinas que abordan el funcionamiento cerebral para explicar el comportamiento humano y las funciones cognitivas en términos de activación de redes neuronales.

"Es el conjunto de ciencias, disciplinas científicas que estudian las funciones químicas, farmacológicas, y patológicas del Sistema Nervioso para comprender con ello las bases biológicas de la conducta humana" (Malfitano Cayuela y Scinica, 2016, pág. 124).

Implica un abordaje interdisciplinario, reconociendo la incapacidad para aprehender 
la complejidad de tal fenómeno desde una óptica única, así como también la necesidad de lograr un vocabulario común para el intercambio e integración de aportes enriquecedores.

En las últimas décadas no solo han sido notables los avances en el conocimiento del funcionamiento cerebral, profundizando aspectos que venían siendo desatendidos por la incapacidad tecnológica de abordarlos, sino que ha crecido enormemente la aplicación del mismo conocimiento tanto en términos cuantitativos como cualitativos. Las Neurociencias han entrado en los más diversos ámbitos incluyendo el de las Ciencias Económicas.

"La utilización de los conocimientos de las Neurociencias aplicados a las Ciencias Económicas podrían contribuir a mejorar las relaciones empáticas de las personas en las organizaciones y de ellas con el ambiente" (Malfitano Cayuela, 2013, pág. 18). "Una vez que se hubo comprendido el modo en que funciona la mente, el siguiente paso es discutir qué influencia debería tener ese conocimiento en materia de gestión" (Tetaz, 2014, pág. 40).

Para los gerentes, las Neurociencias proveen importantes conocimientos y herramientas, que implican:

- mayor comprensión de ellos mismos como decisores, mejorando sus capacidades de planificación y toma de decisiones en general,

- mejor relacionamiento con el otro, optimizando su capacidad negociadora con pares,

- mejor conocimiento de su mercado meta, brindando herramientas para atraer y fidelizar a sus consumidores,

- mayor comprensión de sus recursos humanos, de sus capacidades, su proceso motivacional, lo que permite una mejor gestión de los mismos.

Las neurociencias aplicadas constituyen la llave maestra para la innovación en materia de liderazgo, conducción y gestión de organizaciones y empresas. En el siglo XXI, las nuevas herramientas no están lejos ni fuera de nosotros mismos, sino adentro, en el infinito potencial de nuestro cerebro, en los neurocircuitos que alimentan la toma de decisiones y la inteligencia organizacional (Braidot, 2014, pág. 21).

Un aspecto fundamental en la gestión de los recursos humanos es entender qué los motiva. Esto permitiría ofrecer los incentivos acordes, consiguiendo para el empleador mejorar el desempeño del personal a su cargo, para el empleado aumentar su satisfacción, y para la empresa mejorar el clima laboral en general.

La motivación, en términos generales, puede definirse como el complejo proceso subjetivo que determina el comportamiento humano. Toda conducta es motivada. No hay comportamiento que no resulte de algún nivel de procesamiento cognitivo/ afectivo. Puede ser casi automático e inconsciente o sumamente complejo y con gran desgaste, pero en cualquier caso está presente.

Así definida, la motivación es un proceso incontrolable incluso para el propio sujeto, mucho más para un tercero. Como gerente o supervisor no se puede controlar la motivación de los/as empleados/as. Ahora bien, es posible ejercer influencia en el comportamiento ajeno, aportando los condicionantes apropiados, aunque teniendo 
siempre en cuenta que el resultado final dependerá siempre de cómo el sujeto procese tal estimulación. Se define entonces a la incentivación como la actividad complementaria, por parte de terceros, proveyendo estímulos para ejercer influencia sobre el proceso motivacional.

Sabiendo que se cuenta con distintas capacidades de los/as trabajadores/as, Aquino, Vola-Luhrs, Arecco y Aquino (2010) plantean: "La motivación es el catalizador que pone a estas capacidades en juego, traduciendo la potencialidad interna en realidad externa" (pág. 4).

Desde sus inicios a fines del siglo XIX la Ciencia Administrativa se preocupó por conocer los factores que influyen en la motivación del personal. (...) Actualmente, se habla de la concepción del Hombre Complejo, que destaca que las teorías anteriores no son sustitutas sino más bien complementarias. (...) Sin embargo, se reconoce que cuando se habla de motivación no existe la universalidad y, por lo tanto, cada individuo será motivado de distinta manera. Las Neurociencias contribuyen en el campo de la motivación permitiendo entender y conocer de manera exacta, a través de escaneos cerebrales, la verdadera incidencia de los factores de motivación del individuo. (...) Distintos incentivos pueden activar el neurocircuito de la recompensa, aunque ni siquiera seamos conscientes de que esperamos dicha recompensa (Brain Decision Braidot Center, 2009, pág. 3).

Barthelemy y Rodríguez (2015), en su repaso por el impacto de las Neurociencias en la Administración, retoman la respuesta de Rosler ante la pregunta de cómo se puede motivar a los empleados en el ámbito laboral, quien propone que existen tres tipos de motivación.

La motivación 1.0, que es cumplir con las necesidades básicas. La motivación 2.0, que son las recompensas y castigos que el medio ambiente nos entrega por comportarnos de ciertas formas. La motivación 3.0, o intrínseca, que es aquella que proviene por la gratificación generada en el desempeño de una tarea en sí misma. (...) La diferencia, de lo allí planteado, con el clásico y fenomenal aporte hecho a la teoría de la administración por la pirámide de Maslow, es mínima, por lo menos en la base y en la cúspide de la misma (Barthelemy y Rodríguez, 2015, pág. 48).

"Partiendo de los conocimientos de las neurociencias cognitivas, es posible mejorar la comprensión de los mecanismos motivacionales en la conducta de las personas. El proceso de motivación responde a un concepto denominado arousal, que es la base psicológica que hace que estemos atentos a determinadas cosas. En términos cognitivos, la motivación está relacionada con los objetivos a corto plazo y el reward (recompensa) que estos le brindan al cerebro" (Braidot, 2005, pág. 11).

Es en este contexto que se propone el término Neuroincentivación.

Aprovechar los avances en el conocimiento del comportamiento humano para mejorar el rendimiento del personal y los resultados de la empresa como un todo es una posibilidad al alcance de cualquier empresario/a: no depende tanto del tamaño o los recursos disponibles como de una cuestión de apertura mental de quien toma decisiones. Por el contrario, en las empresas medianas y pequeñas, la mayor cercanía y confianza entre directivos/as y trabajadores/as serían un punto a favor a la hora de articular el conocimiento teórico con el conocimiento directo de los/as 
trabajadores/as particulares y lograr resultados.

\section{Metodología}

Se realizó un estudio descriptivo para a partir de sus resultados formular propuestas de intervención. El mismo fue transversal, de corte cuantitativo.

Se trabajó en dos PyMEs industriales de la ciudad de Rosario. No se tomó una muestra de los empleados de las mismas, sino que se censó a la totalidad del nivel operativo, realizando 30 encuestas en la Empresa A y 43 en la Empresa B (distribuidas en dos plantas).

De los/as 30 empleados/as de la Empresa A, el 56,7\% son varones. El 46,7\% tiene entre 36 y 45 años, el 26,7\% tiene entre 26 y 35 años, 13,3\% tiene hasta 25 años e igual proporción corresponde a los mayores de 45 años. En cuanto a la antigüedad en la empresa, 40\% tiene hasta 5 años, 30\% tiene entre 6 y 10 años, y el 30\% restante lleva más de 10 años en la empresa. En relación al nivel educativo, el 53,3\% tiene nivel primario como máximo, alcanzando el 46,7\% restante el secundario.

En la Empresa B, en cambio, la proporción de varones es de 46,5\%, siendo mayoría las mujeres. Tiene además un plantel bastante más joven: $67,4 \%$ tiene hasta 35 años y solo un $11,6 \%$ supera los 45 años. Así también, la antigüedad es bastante menor: el $60,4 \%$ tiene hasta 5 años en la empresa y solo el 20,9\% supera los 10 años de antigüedad. Por último, tiene también un nivel ligeramente mayor de instrucción, en tanto el $51,2 \%$ cuenta con nivel secundario completo y hay un empleado con nivel terciario.

Se aplicó una encuesta auto administrada, altamente estructurada, previa explicación de las consignas por parte de integrantes del equipo investigador, disponibles para cualquier consulta durante la administración.

El cuestionario fue diseñado ad hoc para esta investigación. Se evaluó su validez de contenido contrastando los reactivos propuestos con las dimensiones normalmente resaltadas en la bibliografía sobre el tema y otros instrumentos de medición del clima organizacional. Así, el clima organizacional fue evaluado por medio de escalas tipo Likert, en función de 97 afirmaciones, valoradas de 1 a 5 en función del nivel de acuerdo. Estas afirmaciones fueron agrupadas en 5 dimensiones:

- Relaciones dentro de la Empresa;

- Condiciones de Trabajo;

- Formación y Evaluación;

- Comunicación; y

- Satisfacción en el Puesto de Trabajo.

No se trabajó con reactivos en negativo y se dio a todas las afirmaciones el mismo valor. Así, para cada afirmación se obtuvo una puntuación por simple promedio de las valoraciones individuales de los/as empleados/as. Para cada dimensión, el puntaje se obtuvo promediando las puntuaciones promedio de cada afirmación contenida en la misma. Para la evaluación global, se calculó el promedio por empleado/a de las 97 afirmaciones, para luego calcular el promedio por empresa a partir de los puntajes individuales. 


\section{Resultados de la Encuesta}

En términos generales, la evaluación global del clima organizacional, promediando las puntuaciones obtenidas en cada una de las afirmaciones, es positiva en ambas empresas, si bien es más alto en una de ellas.

\section{Gráfico 1. Clima organizacional. Evolución global}

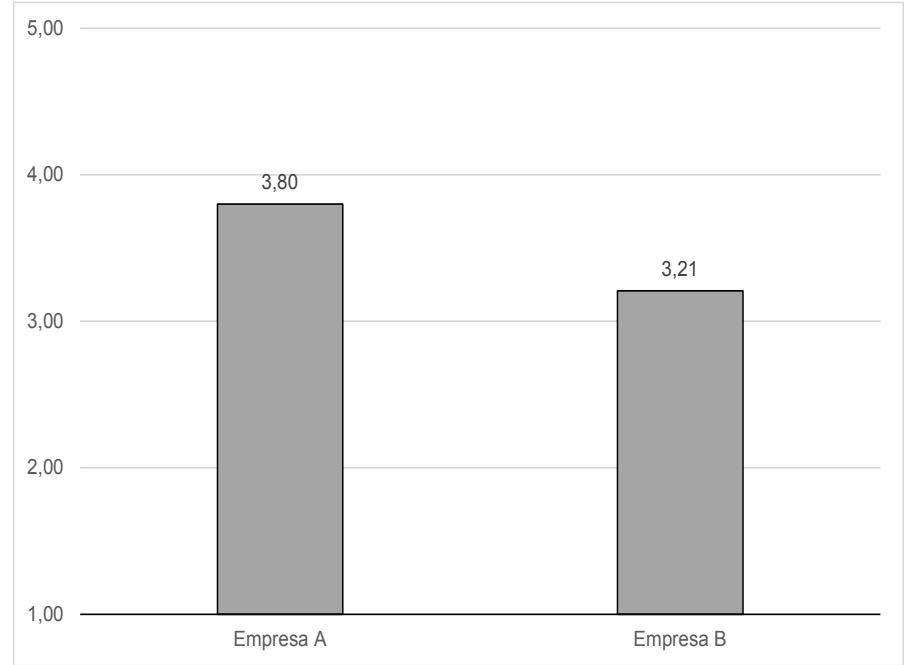

Fuente: Elaboración propia a partir del relevamiento realizado.

En la Empresa A, da un puntaje de 3,80, que daría cuenta de un buen clima organizacional, aunque con suficiente espacio para mejorar. Solo 3 de los/as empleados/as operativos/as encuestados/as (10\%) dan una valoración promedio por debajo del punto medio (3), mientras que son $5(16,7 \%)$ los/as que dan una valoración promedio por encima de 4,50 . La mediana es 3,90 . El $50 \%$ de las valoraciones promedio se ubica entre 3 y 4 puntos.

En la empresa B, en cambio, la puntuación promedio es de 3,21, apenas positiva, dando cuenta de un clima organizacional que no llega a ser malo, pero que apenas cubre las expectativas básicas. Ahora bien, si se analizan los resultados discriminados por planta, se observan diferencias significativas, que ilustrarían dos climas totalmente distintos. En una de estas plantas (Planta B1), el promedio es de 2,56, indicando un clima organizacional bastante malo, mientras que en la otra (Planta B2), el promedio asciende a 4,05. Así, lo que a simple vista parecía una evaluación casi neutral del clima organizacional, en realidad es la compensación entre dos valoraciones opuestas por tener las plantas climas totalmente distintos.

Pasando a la evaluación por dimensiones, existe coincidencia entre ambas empresas respecto a las dimensiones mejor y peor puntuadas en promedio. 
Gráfico 2. Clima organizacional. Evaluación por dimensiones.

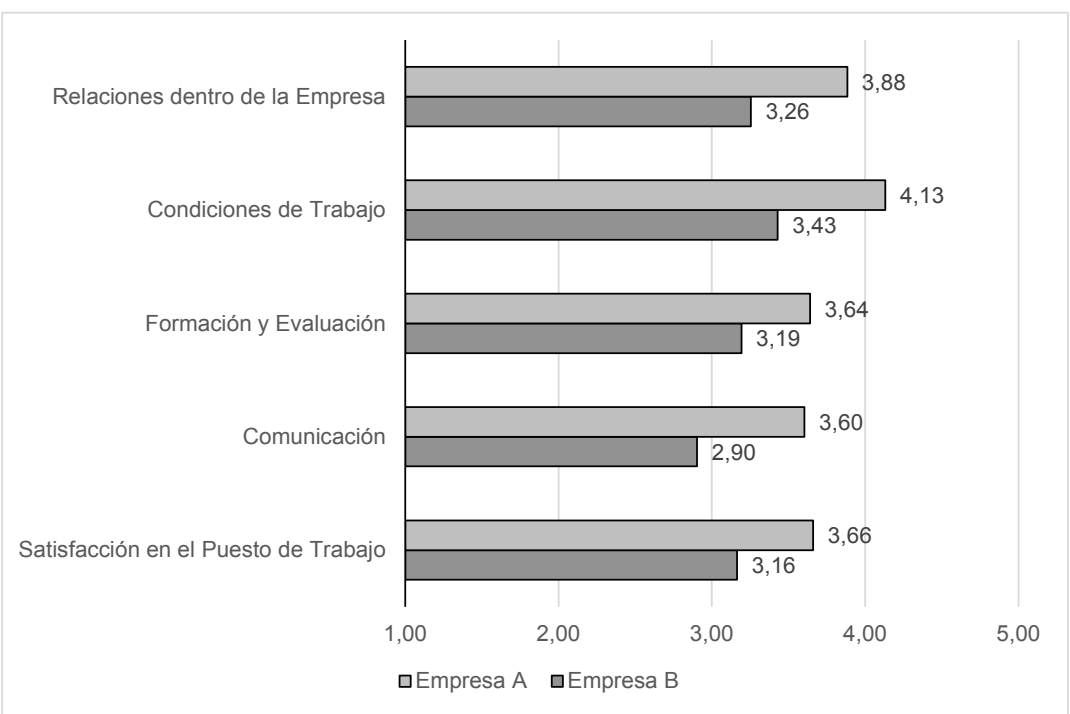

Fuente: Elaboración propia a partir del relevamiento realizado.

En ambas empresas, la puntuación promedio más alta corresponde a las Condiciones de Trabajo (4,13 en la Empresa A y 3,43 en la Empresa B). Esta dimensión se refiere a cuestiones físicas de las instalaciones, horarios y seguridad. Esto es, a las cuestiones más "objetivas", materiales, de la experiencia laboral.

Por el contrario, la dimensión con menor puntaje promedio en ambas empresas corresponde a la Comunicación (3,60 en la Empresa A y 2,90 en la Empresa B). Aquí, el foco está en la transmisión de información, tanto formal como informal, vertical u horizontal.

En cuanto a las dimensiones restantes, la segunda mejor puntuada en ambas empresas es la referida a las Relaciones dentro de la Empresa (3,88 en la Empresa A y 3,26 en la Empresa B). La Satisfacción en el Puesto de Trabajo queda en el tercer lugar para la Empresa $A(3,66)$, mientras que en la Empresa $B$ queda relegado al cuarto $(3,16)$. A la inversa, la Formación y Evaluación ocupa el cuarto lugar para la Empresa $A(3,64)$, mientras que en la Empresa B se ubica como tercera dimensión mejor puntuada $(3,19)$.

En cuanto a las afirmaciones particulares, en la Empresa A son cinco las que obtienen puntuaciones promedio de 4,50 o más:

- "Los baños son suficientes" $(4,70)$.

- "Soy completamente capaz de ejecutar mis tareas actuales" $(4,63)$.

- "Las condiciones de iluminación me permiten desempeñar mi trabajo con normalidad" $(4,53)$.

- "Conozco claramente los resultados que debo lograr con mi trabajo" $(4,50)$.

- "Mantengo una buena relación con mi responsable directo" $(4,50)$.

Dos de estas afirmaciones corresponden a la dimensión Condiciones de Trabajo. Dos corresponden a Formación y Evaluación. La última corresponde a Relaciones 
dentro de la Empresa.

Gráfico 3. Afirmaciones mejor puntuadas. Empresa A.

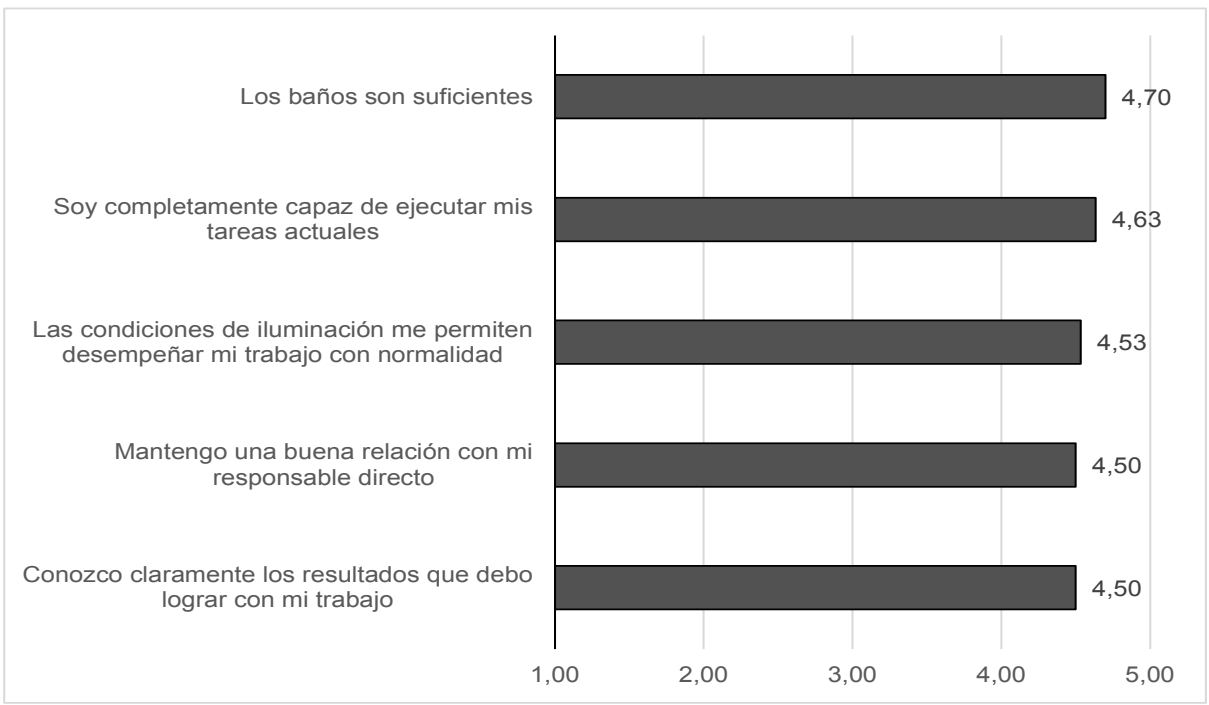

Fuente: Elaboración propia a partir del relevamiento realizado.

En la Empresa $B$, en cambio, solo una afirmación logra obtener una puntuación promedio de 4,50 o más: "Soy completamente capaz de ejecutar mis tareas actuales". Le siguen, con puntajes iguales o superiores a 4 puntos:

- "Tengo claro cuáles son mis tareas y responsabilidades" $(4,44)$.

- "Conozco claramente los resultados que debo lograr con mi trabajo" $(4,43)$.

- "Dispongo de los conocimientos necesarios para realizar mi trabajo con calidad" $(4,21)$.

- "Las condiciones de iluminación me permiten desempeñar mi trabajo con normalidad" $(4,17)$.

- "Estoy dispuesto a realizar un esfuerzo extra para que mi trabajo facilite el de mis compañeros" $(4,07)$.

- "Tengo autonomía para llevar a cabo mi trabajo" $(4,00)$.

Tres de estas afirmaciones corresponden a la dimensión Formación y Evaluación. Dos corresponden a Condiciones de Trabajo. Las otras dos corresponden a Condiciones de Trabajo y Relaciones dentro de la Empresa. 


\section{Gráfico 4. Afirmaciones mejor puntuadas. Empresa B.}

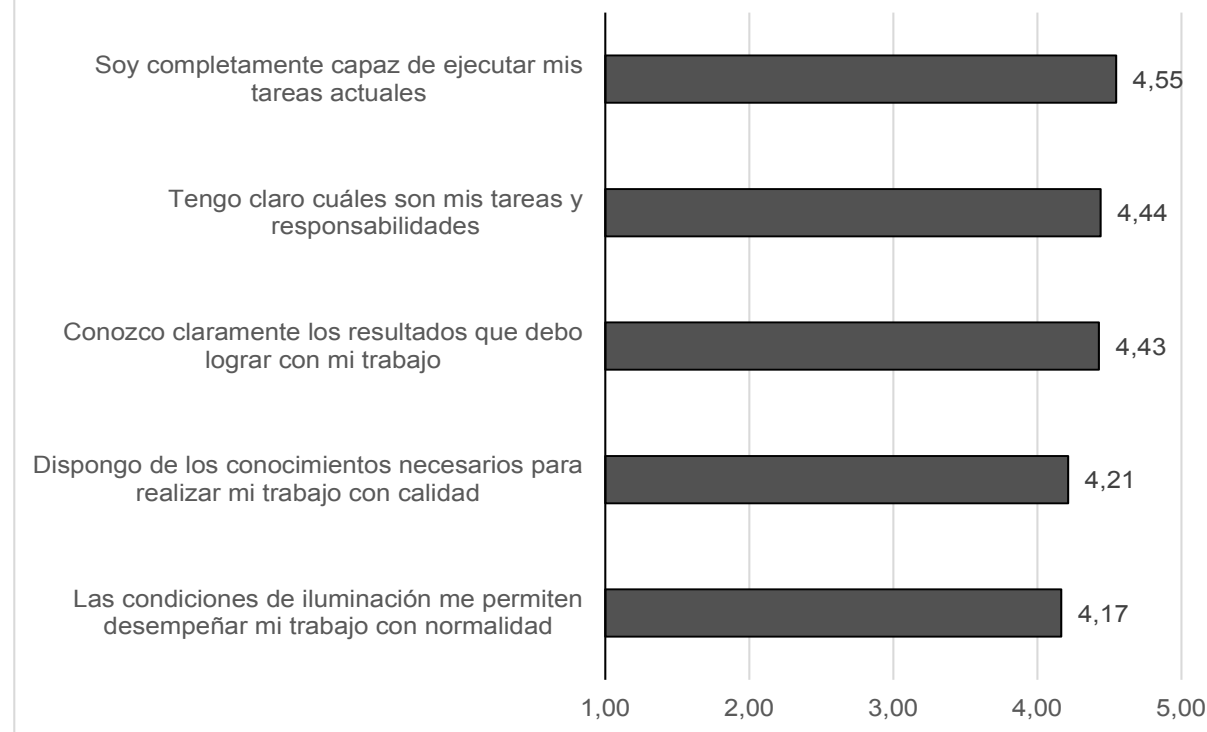

Fuente: Elaboración propia a partir del relevamiento realizado.

Nótese que hay tres afirmaciones que coinciden entre las mejor puntuadas en ambas empresas:

- "Soy completamente capaz de ejecutar mis tareas actuales".

- "Conozco claramente los resultados que debo lograr con mi trabajo".

- "Las condiciones de iluminación me permiten desempeñar mi trabajo con normalidad".

Evidentemente, la cuestión de la iluminación es satisfactoria en ambas empresas; pero lo más importante es la claridad de rol que tienen los/as operarios/as también en ambas: saben lo que se espera de ellos/as y se consideran capacitados/as para hacerlo.

En cuanto a las afirmaciones menos puntuadas, en la Empresa A son cuatro las que obtienen una puntuación promedio por debajo de 3 , que sería el punto medio de acuerdo:

- "Me capacito fuera de la empresa para acceder a los cambios tecnológicos que demanda mi puesto de trabajo" $(2,85)$.

- "Me pagan lo suficiente por lo que hago" $(2,87)$.

- "Tengo la categoría que corresponde en comparación con otras personas que realizan el mismo trabajo" $(2,97)$.

- "Tengo disponible información sobre los puestos vacantes en la Empresa" $(2,97)$. Dos de ellas corresponden a la dimensión Satisfacción en el Puesto de Trabajo y las otras dos a Formación y Evaluación. 
Gráfico 5. Afirmaciones peor puntuadas. Empresa A.

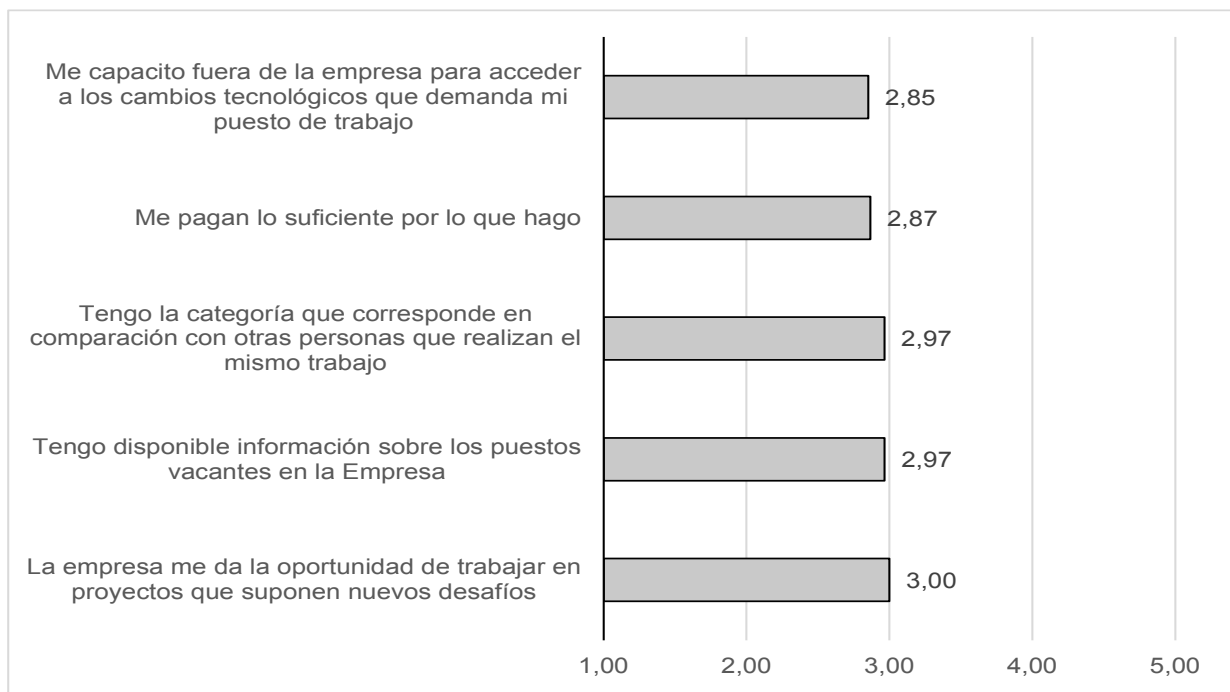

Fuente: Elaboración propia a partir del relevamiento realizado.

Por parte de la Empresa B, son cinco las afirmaciones que obtienen una puntuación promedio por debajo de 2,50; mostrando que hay un importante desacuerdo con las mismas:

- "Me pagan lo suficiente por lo que hago" $(2,21)$.

- "Tengo la categoría que corresponde en comparación con otras personas que realizan el mismo trabajo" $(2,40)$.

- "Recibo información sobre la organización y la evolución de la empresa" $(2,40)$.

- "La Dirección escucha las sugerencias, consejos y dudas de los empleados" $(2,43)$.

- "Las promociones internas se realizan de manera justa" $(2,49)$.

Dos de ellas corresponden a la dimensión Satisfacción en el Puesto de Trabajo, dos a Comunicación y la restante a Formación y Evaluación.

De las 97 afirmaciones evaluadas, son 33 en total las que obtienen un puntaje promedio inferior a 3 en la Empresa B. 


\section{Gráfico 6. Afirmaciones peor puntuadas. Empresa B.}

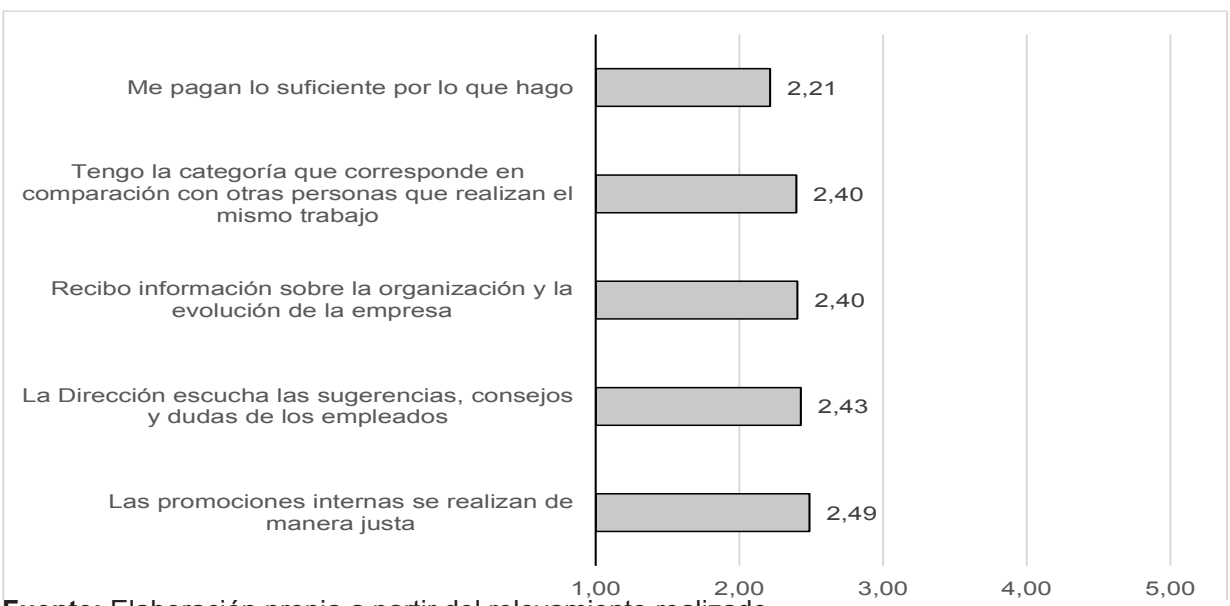

Fuente: Elaboración propia a partir del relevamiento realizado.

Comparando ambas empresas, son dos afirmaciones las que coinciden entre las peor puntuadas:

- "Me pagan lo suficiente por lo que hago".

- "Tengo la categoría que corresponde en comparación con otras personas que realizan el mismo trabajo".

La primera afirmación podría considerarse un reclamo general de la economía actual, en que es común la insatisfacción con el nivel de ingresos, pero articulada con la segunda muestra una situación más compleja que el simple reclamo por mayor salario. Implica cierta insatisfacción con el reconocimiento recibido en términos generales.

Como ya se mencionó, los/as empleados/as consideran saber claramente lo que se espera de ellos/as y estar capacitados/as para lograrlo; aquí se suma que no se sienten suficientemente reconocidos/as por tal esfuerzo, ni en lo económico ni en cuanto a la categoría correspondiente a su nivel de calificación.

A estas afirmaciones puede sumarse una tercera ("Recibo información sobre la organización y la evolución de la empresa"), que si bien en la Empresa A obtiene una puntación por encima de 3 puntos $(3,17)$ se encuentra en el séptimo lugar entre las peor puntuadas. Esta frase se relaciona a cuestiones de transparencia y mayor participación, que en las últimas décadas han venido difundiéndose bastante. Aquí entonces podría pensarse que este desconocimiento de la situación actual de la empresa puede generar ciertas suspicacias respecto a no estar recibiendo un ingreso acorde al (supuesto) éxito comercial.

En el Anexo se presentan las valoraciones promedio para cada una de las 97 afirmaciones en las dos empresas, agrupadas en función de las mencionadas dimensiones, en el orden en que fueron presentadas en el cuestionario.

\section{Conclusiones del Estudio}

Las empresas en estudio presentan bastantes coincidencias respecto al clima 
organizacional y las dimensiones evaluadas. De hecho, hay mayor coincidencia en las evaluaciones globales de las empresas que hacia adentro de la Empresa B, entre sus dos plantas. Aun así, más allá de la mayor satisfacción en la Planta B2, existe igualmente bastante coincidencia en cuanto al orden de los factores evaluados esto es, las afirmaciones mejor y peor puntuadas.

La Empresa A presenta un buen clima organizacional, lo cual da un punto de partida sólido para la aplicación de neuroincentivos que pudieran mejorar la motivación y el compromiso. Los/as empleados/as se muestran en general satisfechos/as con los aspectos intrínsecos del trabajo (el contenido de las tareas realizadas). En cuanto a lo extrínseco, dejando de lado el salario, las mayores debilidades del ambiente laboral parecieran pasar por la relación entre pares, que si bien es buena resulta bastante individualista, sin suficiente integración. Las mayores fortalezas del clima organizacional de la Empresa A parecieran estar en la relación con los superiores y el ambiente físico.

La Empresa B posee dos plantas con climas prácticamente opuestos, mostrándose la Planta B1 como bastante insatisfecha con la situación actual, mientras que la Planta B2 se muestra altamente satisfecha. Esta situación plantea el desafío de identificar las razones que originan estas divergencias con el fin de revertir la insatisfacción reinante en la Planta B1, equiparando el nivel de ambas plantas.

De nuevo, dejando de lado los habituales reclamos de mejoras e incentivos económicos, las quejas recurrentes tienen más relación con los modelos de gestión que apuntan a mejorar la productividad olvidando que se está en relación con sujetos, con intereses y demandas más allá del cumplimiento de las condiciones básicas de higiene y seguridad o recibir sus remuneraciones en tiempo y forma.

Hay una idea general de que lo que quieren los/as empleados/as es "ganar más". Y lo quieren, pero también quieren lo intangible. Quieren reconocimiento, gratitud. Se trata de alimentar al hemisferio derecho del cerebro, que trabaja a su ritmo. Es necesario trabajar en la relación, concentrándose menos en el aspecto de intercambio y más en el "humano".

En esta empresa, en cuanto a factores extrínsecos, resulta necesario fortalecer las relaciones interpersonales, promoviendo el trabajo en equipo, pero sobre todo se hace necesario reforzar la motivación intrínseca: aumentar el interés en lo que se hace y el sentimiento de pertenencia.

Los resultados de esta encuesta permiten comenzar a conocer cómo piensan los empleados y qué los motiva para luego diseñar propuestas concretas que mejoren la capacitación y el desempeño laboral. Ésta tarea se llevará a cabo en la próxima etapa de la investigación, mediante intervenciones que apunten a estimular las funciones del cerebro para revertir las deficiencias encontradas, analizando el impacto de las mismas en los indicadores de productividad, efectividad, calidad y ausentismo.

\section{Propuestas de Neuroincentivos}

A menudo las personas con el paso del tiempo no tienen el mismo compromiso con el trabajo que alguna vez tuvieron y el mismo resulta ser "solo un trabajo" importándole realizar solo lo mínimo indispensable, nada los incentiva a hacer algo más. Sin 
embargo, el solo hecho de que sigan en ese trabajo implica que existe compromiso. Lo que hace falta es despertarlo.

Una persona con compromiso es alguien a quien le importa lo que hace. No se limita a cumplir, sino que hace un esfuerzo mayor. Tiene en la cabeza lo que le importa, y quiere que su tarea esté bien hecha. Se exige más allá de lo que es exigida. El desafío es identificar los intereses, fortalezas y talentos de las personas y alinearlos con sus asignaciones de trabajo. Un/a empleado/a mal asignado/a, que no utiliza bien sus habilidades, se adaptará y con el paso del tiempo esto generará estrés.

Por el contrario, si están bien asignados/as, estarán más enfocados/as en su tarea, en lo que Csikszentmihalyi (2003) llama "fluir" y se refiere a un estado mental en el cual la persona está completamente inmersa en su tarea, totalmente implicada, dando como resultado una mejora en la productividad.

El ambiente de trabajo debe permitir que los/as empleados/as se sientan cómodos/ as y reconocidos/as, ya que el estrés que genera el temor sobre su estabilidad laboral impide que trabaje el neocórtex que es el encargado del pensamiento lógico y la consciencia, cuya función no es responder de manera previsible a estímulos, sino que, a partir de conocimientos ya procesados, improvisa respuestas originales; es el que permite desarrollar la creatividad e innovación.

Para comenzar con la aplicación de los neuroincentivos que permitan mejorar la motivación y el compromiso, se plantea analizar las cinco dimensiones establecidas en la encuesta, a saber: Condiciones de Trabajo, Relaciones dentro de la Empresa, Satisfacción en el Puesto de Trabajo, Formación y Evaluación, y finalmente Comunicación. Una vez definida la combinación de herramientas, se comenzará a perfilar la implementación con la consulta pertinente a los titulares de la firma.

Se empezó a trabajar en cada ítem, desarrollando un brainstorming con los/as integrantes del grupo, y del mismo surgieron diferentes alternativas de aplicación en las dimensiones, que podrían ser de utilidad en los ensayos a realizar. Estos resultados preliminares se relatan a continuación:

Condiciones de trabajo: En este aspecto, en esta empresa se respetan a rajatabla las condiciones de trabajo impuestas por las regulaciones laborales en cuanto a elementos de seguridad personal (botines, protectores auditivos, fajas lumbares, lentes, etc.) y del ambiente (matafuegos, protectores en máquinas, líneas de circulación, etc.). Se dan también las prohibiciones clásicas como el uso del celular o escuchar música con auriculares. Estas circunstancias, si bien son estrictamente legales y entendibles, en ocasiones se extreman al punto de ignorar que las personas que trabajan necesitan ser consideradas como tales, y no ser confundidas con máquinas en su desempeño. Con pequeños gestos y sin dejar de respetas las regulaciones establecidas, podrían mejorarse sustancialmente esas condiciones de trabajo. Entre otras propuestas, se sugiere: a) colocar pizarrones que permitan al personal indicar sugerencias, noticias, frases para reflexionar, chistes, etc.; b) implementar una suerte de concurso relacionado con el punto anterior; c) implementar música apropiada para trabajar (que sirva para relajación, por ejemplo); d) realizar antes de empezar el turno o al finalizar el mismo, en forma voluntaria, cinco minutos de ejercicios de respiración dirigidos o mindfulness. Tener momentos de descanso puede lograr una nueva 
perspectiva para resolver problemas, moverse periódicamente ayuda a activar el cerebro logrando la producción de neurotransmisores que favorecen el aprendizaje, la socialización, la serenidad, etc.

Relaciones dentro de la Empresa: En este punto, se considera imprescindible, como ya fue mencionado, fortalecer y mejorar las relaciones interpersonales, promoviendo el trabajo en equipo; y para cumplir con este reto, es fundamental la capacitación. Se sugiere implementar cursos y talleres, impartidos por profesionales externos, pero no se descarta su implementación guiada a manos del propio personal (previa capacitación del mismo), recordando que "enseñar es aprender dos veces". Esta frase sería de gran utilidad en este caso.

Otro medio para reforzar las relaciones son las actividades lúdicas extra laborales, tales como reuniones con o sin motivos específicos (fútbol, clases abiertas de yoga o pilates, clases de baile, etc.) con finales culinarios (asados, pizzas o hamburguesas) que recuperan el clima de cordialidad y coadyuvan al sentimiento de pertenencia. Promover la necesidad de compartir y comunicar el conocimiento. A medida que se "corra la voz", esto genera una onda expansiva que permite contagiar a los/as indecisos/as y aun a los/as pesimistas. En este momento, debe individualizarse a las personas que motiven y sean disparadores de estas iniciativas.

Satisfacción en el puesto de trabajo: Esta es quizás la dimensión que plantea el mayor desafío en cuanto a la aplicación y evaluación de los neuroincentivos. Es también la de trabajo más exhaustivo para el grupo de investigación. Se buscarán neuroincentivos tales como: chocolate, colores, música, uso del factor sorpresa. Para ello, se consultará a los titulares y supervisores a fin de indagar sobre la posibilidad de sacar a la gente de su zona de confort y enfrentarla a nuevos desafíos que enriquezcan sus puestos de trabajo. Se intentará promover la felicidad de la persona, dentro y fuera del trabajo, mediante la entrega de herramientas y tips de autoayuda, que les permitan mejorar pequeños aspectos de su vida cotidiana (tanto en lo laboral como en lo personal).

Formación y evaluación: Este aspecto es esencial en este trabajo de investigación. Se propone realizar intensos programas de capacitación, para formar al personal, tanto en los aspectos técnicos como otros relacionados con la formación de equipos de trabajo. Para plantearlo en términos de neurociencia, para aprehender, hace falta motivación y esfuerzo; esto produce dopamina, que reduce la resistencia al cambio. Dentro de las herramientas de capacitación la repetición ayuda a la formación de redes neuronales.

Comunicación: Para concluir, es necesario informar y solo se consigue comunicando claramente los objetivos que se quieren alcanzar. Evitar las ambigüedades y ser lo suficientemente claros, permitirá incentivar y "reclutar voluntades"; comunicar con frases cortas ayuda a mantener la atención del sistema reticular. El trabajo exhaustivo, se dará especialmente con los supervisores y mandos medios, a quienes se capacitará en herramientas de comunicación. La comunicación es $10 \%$ lo que se dice y $90 \%$ cómo se dice. Se debe forjar una buena comunicación para que llegue a buen puerto el mensaje que se quiere transmitir.

\section{Consideraciones finales}


En el punto anterior se presentaron algunos de los pilares propuestos en una primera etapa experimental.

Se están midiendo indicadores. Se tiene una idea acabada del punto de partida, y también se definen las propuestas alternativas para llevar adelante los objetivos planteados al iniciar esta investigación.

Estas intervenciones apelan a fortalecer las conexiones neuronales recurriendo a herramientas auditivas y kinestésicas, entre otras, que hagan más agradables las condiciones de trabajo; favorecer la creatividad reduciendo la incertidumbre y evitando el estrés; despertar el interés por la tarea fomentando la participación y resaltando los logros personales, buscando así colaborar con la satisfacción en el puesto de trabajo e influyendo en la motivación intrínseca.

Por otra parte se intentará trabajar sobre la empatía, tanto entre pares como entre los distintos niveles, reforzando el espíritu de equipo, realizando reuniones de trabajo y actividades extra laborales, tendiendo a consolidar las relaciones dentro de la empresa que es otro de los puntos débiles del clima laboral que tienen en común estas organizaciones. La relación con el nivel de supervisión es fundamental, es un nexo indispensable para la aplicación de neuroincentivos entre el personal operativo, razón por la cual se lo capacitará expresamente en esa dirección dentro de las previsiones de formación y capacitación, así como también en herramientas de comunicación que hagan llegar el mensaje de manera más efectiva al cerebro aumentando el interés y mejorando la comprensión.

Se espera beneficiar a los/as empleados/as realzando la calidad de su trabajo personal, y también a la empresa corrigiendo el bajo desempeño de algunos indicadores como ausentismo y productividad

El tiempo y el arduo trabajo ya realizado y el que queda por venir, permitirán continuar con este relato. Se espera sea un buen aporte para las PyMEs de la región Rosario y el equipo de investigación se compromete a entregar el mejor esfuerzo para que ello se cristalice a la brevedad.

\section{Referencias bibliográficas}

Albano, S., Oviedo, R., Santero, M., Sassone, M. y Martín, S. (2016). Desarrollo de una metodología de aplicación de incentivos basada en neurociencias. En Actas Vigesimoprimeras Jornadas "Investigaciones en la Facultad" de Ciencias Económicas y Estadística. Universidad Nacional de Rosario. Rosario, Argentina.

Aquino, J., Vola-Luhrs, R., Arecco, M. y Aquino, G. (2010). Recursos Humanos. $4^{\text {a }}$ edición. Buenos Aires, Argentina: Prentice Hall-Pearson Educación.

Barthelemy, H. y Rodríguez, V. (2015). Las neurociencias y su impacto en la Administración. En II Congreso Latinoamericano de Administración y VEncuentro Internacional de Administración de la Región Jesuítico Guaraní. Posadas, Argentina. 
Braidot, N. (2014). Neuromanagement. La revolución neurocientífica en las organizaciones, del management al neuromanagement. Buenos Aires, Argentina: Granica.

Braidot, N. (2005). Neuromarketing. Neuroeconomía y negocios. Madrid, España: Puerto Norte-Sur.

Brain Decision Braidot Center (2009). Neurociencias y RRHH. Aplicaciones. Brain Decision Braidot Center. Recuperado de http://www.econo.unlp.edu.ar/ uploads/docs/e_news_marzo_2009.pdf.

Csikszentmihalyi, M. (2003). Fluir en los Negocios. Recuperado de http://academyw. $\mathrm{com} /$ recursos/mas/Directorio/Recursos/rfwyz/Mas/Lib_mes/Fluir\%20en\%20 los\%20negocios.pdf.

Malfitano Cayuela, O. y Scinica, E. (2016). Neuroestrategia. $1^{\text {a }}$ edición para el profesor. Buenos Aires, Argentina: Alta Gerencia.

Malfitano Cayuela, O. (2013). Neurocompetencias. Un pensamiento sistémico para la reflexión. Revista Alta Gerencia, XIII(62-1). Recuperado de http://www. altagerenciainternacional.com/publicaciones/revista-altangerencial.

Tetaz, M. (2014). Neuroanatomía de la decisión. Revista Alta Gerencia, XIV(63-2). Recuperado de http://www.altagerenciainternacional.com/publicaciones/ revista-alta-gerencial. 
Anexos: Evaluaciones promedio por afirmación

\begin{tabular}{|c|c|c|}
\hline Relaciones dentro de la Empresa & $\begin{array}{c}\text { Empresa } \\
\text { A }\end{array}$ & $\begin{array}{c}\text { Empresa } \\
\text { B }\end{array}$ \\
\hline $\begin{array}{l}\text { Cuento con la colaboración de las personas de otros } \\
\text { departamentos }\end{array}$ & 3,14 & 3,19 \\
\hline $\begin{array}{l}\text { Las personas con las que me relaciono en la empresa } \\
\text { actúan con respeto y de manera ética }\end{array}$ & 3,77 & 3,28 \\
\hline $\begin{array}{l}\text { Mi responsable directo se preocupa por conocer mis } \\
\text { necesidades e intereses }\end{array}$ & 4,03 & 2,98 \\
\hline $\begin{array}{l}\text { Estoy dispuesto a realizar un esfuerzo extra para que } \\
\text { mi trabajo facilite el de mis compañeros }\end{array}$ & 3,53 & 4,07 \\
\hline $\begin{array}{l}\text { Mi responsable directo se preocupa por mantener un } \\
\text { buen clima en el equipo }\end{array}$ & 3,73 & 3,14 \\
\hline Considero que existe un buen ambiente de trabajo & 3,93 & 3,05 \\
\hline $\begin{array}{l}\text { Mantengo una buena relación con mi responsable } \\
\text { directo }\end{array}$ & 4,50 & 3,63 \\
\hline Los empleados confían en sus colegas & 3,52 & 2,66 \\
\hline $\begin{array}{l}\text { Cuento con la colaboración de mis compañeros de } \\
\text { departamento }\end{array}$ & 3,43 & 3,42 \\
\hline $\begin{array}{l}\text { Mi responsable directo respeta las diferencias de } \\
\text { cultura, sexo, religión... }\end{array}$ & 4,13 & 3,27 \\
\hline Los empleados tratan a sus colegas con respeto & 3,73 & 3,54 \\
\hline $\begin{array}{l}\text { Las reglas y regulaciones de la empresa son siempre } \\
\text { cumplidas al pie de la letra por la Dirección }\end{array}$ & 3,62 & 2,88 \\
\hline $\begin{array}{l}\text { Los empleados reciben un trato respetuoso de } \\
\text { directivos y gerentes }\end{array}$ & 4,47 & 3,67 \\
\hline $\begin{array}{l}\text { La Dirección alienta a que los empleados trabajen en } \\
\text { equipo }\end{array}$ & 3,60 & 3,09 \\
\hline $\begin{array}{l}\text { La administración se hace responsable de sus } \\
\text { acciones }\end{array}$ & 4,00 & 3,21 \\
\hline Cuando ingresé en la Compañía me sentí bienvenido & 4,31 & 3,71 \\
\hline $\begin{array}{l}\text { Los empleados confían en cualquier decisión tomada } \\
\text { por la Dirección }\end{array}$ & 3,72 & 2,93 \\
\hline $\begin{array}{l}\text { Los directivos y gerentes motivan a los empleados a } \\
\text { cumplir con los plazos }\end{array}$ & 4,00 & 3,17 \\
\hline $\begin{array}{l}\text { Mi responsable directo me trata justamente y evita } \\
\text { cualquier tipo de favoritismos }\end{array}$ & 3,73 & 3,36 \\
\hline $\begin{array}{l}\text { Mi responsable directo se preocupa por transmitir los } \\
\text { valores, misión y objetivos de la empresa }\end{array}$ & 4,14 & 3,17 \\
\hline
\end{tabular}




\begin{tabular}{|l|c|c|}
\hline $\begin{array}{l}\text { Los empleados siempre pueden pedirle consejos o } \\
\text { ayuda a directivos y gerentes }\end{array}$ & 4,13 & 3,09 \\
\hline $\begin{array}{l}\text { Los empleados siempre pueden pedirle consejos o } \\
\text { ayuda a directivos y gerentes }\end{array}$ & 3,83 & 2,79 \\
\hline No existen prácticas discriminatorias en esta empresa & 4,30 & 3,58 \\
\hline
\end{tabular}

\begin{tabular}{|c|c|c|}
\hline Condiciones de Trabajo & $\begin{array}{c}\text { Empresa } \\
\text { A }\end{array}$ & $\begin{array}{c}\text { Empresa } \\
\text { B }\end{array}$ \\
\hline $\begin{array}{l}\text { Las condiciones de espacio me permiten desempeñar } \\
\text { mi trabajo con normalidad }\end{array}$ & 4,37 & 3,36 \\
\hline $\begin{array}{l}\text { Dispongo de los materiales y recursos necesarios para } \\
\text { realizar mi trabajo }\end{array}$ & 4,17 & 3,41 \\
\hline $\begin{array}{l}\text { Desde mi ingreso, la Empresa se ha ido transformando } \\
\text { en un lugar mejor para trabajar }\end{array}$ & 4,34 & 3,26 \\
\hline Estoy conforme con mis horarios de trabajo & 4,17 & 3,74 \\
\hline $\begin{array}{l}\text { La empresa cumple las normas de Seguridad y Salud } \\
\text { en el trabajo }\end{array}$ & 3,73 & 3,20 \\
\hline $\begin{array}{l}\text { Estoy satisfecho con los beneficios sociales que me } \\
\text { ofrece la empresa }\end{array}$ & 3,77 & 3,19 \\
\hline $\begin{array}{l}\text { Las condiciones de orden me permiten desempeñar mi } \\
\text { trabajo con normalidad }\end{array}$ & 4,00 & 3,18 \\
\hline $\begin{array}{l}\text { Tengo todo el tiempo que necesito para realizar mis } \\
\text { tareas actuales }\end{array}$ & 3,97 & 3,17 \\
\hline $\begin{array}{l}\text { Los recursos y equipos de la compañía son } \\
\text { compartidos por todos los empleados }\end{array}$ & 3,93 & 3,43 \\
\hline $\begin{array}{l}\text { Las condiciones de ruido me permiten desempeñar mi } \\
\text { trabajo con normalidad }\end{array}$ & 3,50 & 3,31 \\
\hline Los baños son suficientes & 4,70 & 3,20 \\
\hline Tengo todo el equipamiento que necesito para trabajar & 4,13 & 4,13 \\
\hline Los baños están siempre limpios & 4,40 & 3,12 \\
\hline Me siento cómodo en mi lugar de trabajo & 4,27 & 3,88 \\
\hline Me siento seguro en mi lugar de trabajo & 4,37 & 3,93 \\
\hline $\begin{array}{l}\text { Las condiciones temperatura me permiten desempeñar } \\
\text { mi trabajo con normalidad }\end{array}$ & 3,83 & 3,19 \\
\hline Trabajo solamente las horas previstas & 3,93 & 3,38 \\
\hline $\begin{array}{l}\text { Las condiciones de iluminación me permiten } \\
\text { desempeñar mi trabajo con normalidad }\end{array}$ & 4,53 & 4,17 \\
\hline
\end{tabular}




\begin{tabular}{|l|c|c|}
\hline $\begin{array}{l}\text { Tengo confianza en que mi puesto de trabajo actual es } \\
\text { seguro }\end{array}$ & 4,23 & 3,95 \\
\hline $\begin{array}{l}\text { Todas las herramientas se mantienen de forma } \\
\text { adecuada }\end{array}$ & 4,30 & 4,30 \\
\hline
\end{tabular}

\begin{tabular}{|c|c|c|}
\hline Formación y Evaluación & $\begin{array}{c}\text { Empresa } \\
\text { A }\end{array}$ & $\begin{array}{c}\text { Empresa } \\
\text { B }\end{array}$ \\
\hline $\begin{array}{l}\text { Recibo capacitación para actualizar los conocimientos } \\
\text { de mi trabajo }\end{array}$ & 3,33 & 3,19 \\
\hline $\begin{array}{l}\text { Recibí una preparación apropiada para la posición en } \\
\text { la cual trabajo hoy }\end{array}$ & 3,47 & 3,68 \\
\hline $\begin{array}{l}\text { Soy completamente capaz de ejecutar mis tareas } \\
\text { actuales }\end{array}$ & 4,63 & 4,55 \\
\hline $\begin{array}{l}\text { Me capacito en la empresa para acceder a los cambios } \\
\text { tecnológicos que demanda mi puesto de trabajo }\end{array}$ & 3,62 & 3,17 \\
\hline $\begin{array}{l}\text { Los planes de formación de la empresa se adecuan } \\
\text { a mis necesidades de desarrollo profesional en la } \\
\text { Compañía }\end{array}$ & 3,77 & 3,07 \\
\hline $\begin{array}{l}\text { Conozco claramente los resultados que debo lograr } \\
\text { con mi trabajo }\end{array}$ & 3,07 & 4,43 \\
\hline $\begin{array}{l}\text { Me capacito fuera de la empresa para acceder a los } \\
\text { cambios tecnológicos que demanda mi puesto de } \\
\text { trabajo }\end{array}$ & 2,85 & 2,85 \\
\hline $\begin{array}{l}\text { Dispongo de los conocimientos necesarios para } \\
\text { realizar mi trabajo con calidad }\end{array}$ & 4,07 & 4,21 \\
\hline $\begin{array}{l}\text { Mi responsable me felicita cuando realizo bien mi } \\
\text { trabajo }\end{array}$ & 3,37 & 2,51 \\
\hline $\begin{array}{l}\text { Mi responsable escucha mis opiniones y me hace } \\
\text { partícipe de las decisiones }\end{array}$ & 3,52 & 2,69 \\
\hline $\begin{array}{l}\text { Mi responsable me proporciona periódicamente } \\
\text { información sobre mi desempeño }\end{array}$ & 3,60 & 2,83 \\
\hline Siento que mi desempeño es apreciado por otros & 2,83 & 2,91 \\
\hline $\begin{array}{l}\text { Mi responsable hace un seguimiento de mi crecimiento } \\
\text { laboral }\end{array}$ & 3,67 & 2,76 \\
\hline $\begin{array}{l}\text { Tengo la categoría que corresponde en comparación } \\
\text { con otras personas que realizan el mismo trabajo }\end{array}$ & 2,97 & 2,40 \\
\hline Los empleados son evaluados con precisión & 3,67 & 2,86 \\
\hline
\end{tabular}




\begin{tabular}{|c|c|c|}
\hline Comunicación & $\begin{array}{c}\text { Empresa } \\
\text { A }\end{array}$ & $\begin{array}{c}\text { Empresa } \\
\text { B }\end{array}$ \\
\hline $\begin{array}{l}\text { Tengo disponible información sobre el catálogo de } \\
\text { productos y servicios que ofrece la empresa }\end{array}$ & 3,23 & 2,51 \\
\hline $\begin{array}{l}\text { Mi responsable es claro y específico cuando define mis } \\
\text { objetivos de trabajo o los del departamento }\end{array}$ & 4,17 & 3,26 \\
\hline Recibo la ayuda que necesito de otros sectores & 3,20 & 3,34 \\
\hline $\begin{array}{l}\text { Los comunicados internos me proporcionan } \\
\text { información útil }\end{array}$ & 3,54 & 3,03 \\
\hline $\begin{array}{l}\text { Cuando ingresé en la Empresa recibí suficiente } \\
\text { información sobre la misma }\end{array}$ & 3,70 & 3,07 \\
\hline $\begin{array}{l}\text { Recibo información sobre los elementos que } \\
\text { componen mi salario }\end{array}$ & 3,59 & 2,88 \\
\hline $\begin{array}{l}\text { La comunicación interna en la empresa es una } \\
\text { actividad permanente y planificada }\end{array}$ & 3,69 & 2,83 \\
\hline $\begin{array}{l}\text { Los valores de la compañía son comprendidos y } \\
\text { compartidos por los empleados }\end{array}$ & 3,50 & 2,69 \\
\hline $\begin{array}{l}\text { Recibo información sobre la organización y la evolución } \\
\text { de la empresa }\end{array}$ & 3,17 & 2,40 \\
\hline $\begin{array}{l}\text { Las reglas y regulaciones de la empresa son siempre } \\
\text { cumplidas por los empleados }\end{array}$ & 3,83 & 3,29 \\
\hline $\begin{array}{l}\text { La Dirección escucha las sugerencias, consejos y } \\
\text { dudas de los empleados }\end{array}$ & 3,52 & 2,43 \\
\hline $\begin{array}{l}\text { Al unirme a la Empresa, recibí suficiente información } \\
\text { sobre el área donde trabajo y la función que realizo }\end{array}$ & 3,80 & 3,31 \\
\hline $\begin{array}{l}\text { Se fomenta el diálogo entre empleados y directivos y } \\
\text { gerentes }\end{array}$ & 3,73 & 2,74 \\
\hline $\begin{array}{l}\text { Las metas y la estrategia de la compañía son } \\
\text { explicadas regularmente por los directivos y gerentes }\end{array}$ & 3,80 & 2,86 \\
\hline
\end{tabular}

\begin{tabular}{|l|c|c|}
\hline Satisfacción en el Puesto de Trabajo & $\begin{array}{c}\text { Empresa } \\
\text { A }\end{array}$ & $\begin{array}{c}\text { Empresa } \\
\text { B }\end{array}$ \\
\hline $\begin{array}{l}\text { Pienso que si desempeño bien mi trabajo, tengo } \\
\text { posibilidad de ascender en la empresa }\end{array}$ & 3,24 & 2,93 \\
\hline $\begin{array}{l}\text { Considero adecuados los criterios de evaluación de mi } \\
\text { desempeño en la empresa }\end{array}$ & 3,80 & 3,80 \\
\hline $\begin{array}{l}\text { La empresa me da la oportunidad de trabajar en } \\
\text { proyectos que suponen nuevos desafíos }\end{array}$ & 3,00 & 2,58 \\
\hline
\end{tabular}




\begin{tabular}{|c|c|c|}
\hline $\begin{array}{l}\text { Creo que tengo la oportunidad de desarrollarme } \\
\text { profesionalmente en la empresa }\end{array}$ & 3,37 & 2,98 \\
\hline $\begin{array}{l}\text { Conozco cómo mi trabajo contribuye a conseguir los } \\
\text { resultados de mi departamento }\end{array}$ & 4,14 & 3,90 \\
\hline $\begin{array}{l}\text { Pienso que la empresa es un buen lugar para trabajar } \\
\text { y me gustaría continuar trabajando aquí }\end{array}$ & 4,10 & 3,51 \\
\hline Las promociones internas se realizan de manera justa & 3,40 & 2,49 \\
\hline $\begin{array}{l}\text { Tengo la oportunidad de proponer nuevos proyectos o } \\
\text { nuevas formas de realizar el trabajo }\end{array}$ & 3,53 & 3,53 \\
\hline Tengo autonomía para llevar a cabo mi trabajo & 4,23 & 4,00 \\
\hline $\begin{array}{l}\text { Tengo disponible información sobre los puestos } \\
\text { vacantes en la Empresa }\end{array}$ & 2,97 & 2,88 \\
\hline Me siento orgulloso de trabajar para esta empresa & 4,00 & 3,47 \\
\hline $\begin{array}{l}\text { Al asumir una nueva posición en la empresa, mi } \\
\text { responsable me informa de manera clara sobre las } \\
\text { funciones y responsabilidades del puesto de trabajo }\end{array}$ & 3,56 & 3,35 \\
\hline $\begin{array}{l}\text { Recomiendo a mi empresa como un lugar donde } \\
\text { trabajar }\end{array}$ & 3,93 & 3,30 \\
\hline $\begin{array}{l}\text { Mi capacidad profesional está de acuerdo a las tareas } \\
\text { y responsabilidades asignadas }\end{array}$ & 4,10 & 3,67 \\
\hline $\begin{array}{l}\text { Mi trabajo me ofrece retos y la oportunidad de seguir } \\
\text { mejorando }\end{array}$ & 3,83 & 3,49 \\
\hline $\begin{array}{l}\text { Tengo la información que necesito para realizar mi } \\
\text { trabajo con excelencia }\end{array}$ & 3,80 & 3,41 \\
\hline Mi trabajo es reconocido y valorado & 3,40 & 2,70 \\
\hline $\begin{array}{l}\text { Actualmente estoy satisfecho con mi trabajo en la } \\
\text { empresa }\end{array}$ & 4,07 & 3,86 \\
\hline Tengo claro cuáles son mis tareas y responsabilidades & 4,43 & 4,44 \\
\hline Me siento partícipe del proyecto de la empresa & 3,57 & 2,95 \\
\hline $\begin{array}{l}\text { Se alienta a los empleados a buscar nuevas formas de } \\
\text { resolver problemas }\end{array}$ & 2,95 & 2,52 \\
\hline $\begin{array}{l}\text { La compañía aprecia y valora las ideas de los } \\
\text { empleados }\end{array}$ & 3,60 & 2,56 \\
\hline Me pagan lo suficiente por lo que hago & 2,87 & 2,21 \\
\hline Mis directivos y gerentes monitorean mi éxito & 3,40 & 2,60 \\
\hline Las tareas que realizo me resultan motivadoras & 3,73 & 3,42 \\
\hline
\end{tabular}

Fuente: Elaboración propia a partir del relevamiento realizado. 